\title{
Connexin's auditory connection
}

File name: NRN0406_AR_HL1 Word count: 455 Accompanying picture: YES File name of picture: Nature's anatomy: BS31007 (AA022902)

URLs
Gap junctions - which contain intercellular channels composed of connexin proteins - allow molecules and ions to flow directly between most types of cell, and constitute electrical synapses between neurons. Mutations in connexin genes underlie approximately half of all cases of genetic deafness in childhood, but their modes of action are not well understood. A report in the Journal of Neuroscience provides new insights into the role of a recently identified connexin - CX29 - in auditory perception.

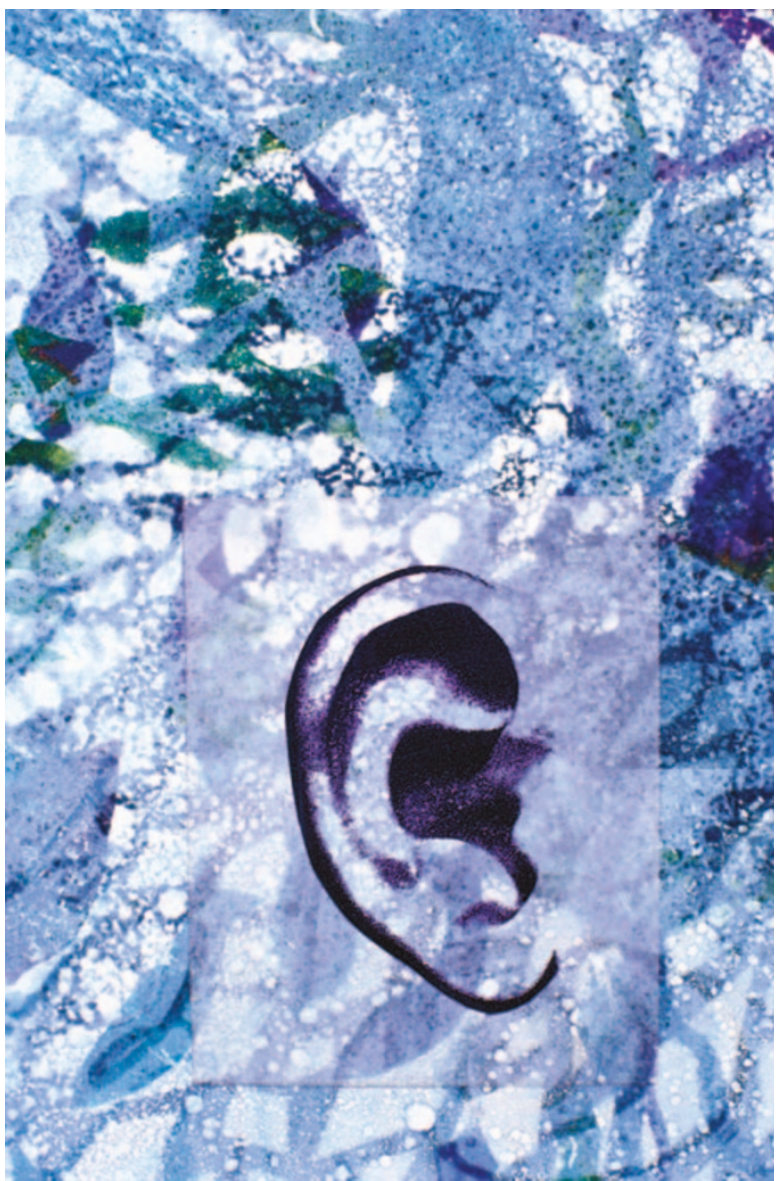

Four types of connexin, including CX29, are expressed in the cochlea. Tang and co-workers found that CX29 is abundantly and exclusively expressed in Schwann cells that myelinate the soma and processes of spiral ganglion neurons, which communicate sound signals transduced by hair cells to the cochlear nuclei in the brain stem. By contrast, the other connexins are primarily expressed in cochlear supporting cells and/or fibrocytes.

To determine the functions of CX29, Tang and colleagues studied mice that were deficient in $C \times 29$. These mice were impaired in several aspects of auditory perception. Specifically, their hearing sensitivity across a range of frequencies was defective. In addition, they had sustained hearing loss for highfrequency sounds in response to stimulation with white noise, which resulted in only temporary impairments in wild-type mice.

The cochlear hair cells of these mice were morphologically normal, so what is the cellular mechanism that underlies these deficits? The presence of high levels of CX29 in Schwann cells suggested that myelination might be affected. Indeed, the myelin around the soma of spiral ganglion neurons was severely disorganized, although axonal myelin was normal.
CX29 is expressed in most Schwann cells and oligodendrocytes, yet only myelin in the spiral ganglion is affected in Cx29-knockout mice. Most myelinating glia express several connexin genes, each of which might compensate for the loss of another. However, these data suggest that spiral ganglion Schwann cells might express only CX29. Therefore, it seems that CX29 makes a unique contribution to myelination in the spiral ganglia and auditory perception.

This elegant study reveals the mechanisms by which CX29 plays its part in normal hearing. Moreover, the pattern of deficits in mice deficient in CX29 was consistent with that seen in patients with auditory neuropathies. Therefore, as the authors point out, these mice could provide a valuable model with which to explore the cellular and molecular basis of auditory neuropathy.

Alison Rowan

ORIGINAL RESEARCH PAPER Tang, W. et al Connexin2 9 is highly expressed in cochlear Schwann cells, and it is required for the normal development and function of the auditory nerve of mice.J. Neurosci. 26, 1991-1999 (2006) FURTHER READING Söhl, G., Maxeiner, S. \& Willecke, K. Expression and functions of neuronal gap junctions. Nature Rev. Neurosci. 6, 191-200 (2005) 